\title{
Nota sobre la última transgresión marina en la costa de Galicia
}

\section{Note on the last marine transgression on the coast of Galicia.}

VIDAL-ROMANÍ, J. R. ${ }^{1}$ y GRANDAL D'ANGLADE, A ${ }^{1}$

${ }^{1}$ Instituto Universitario de Xeoloxía, Universidade da Coruña. ESCI, Campus de Elviña. 15071 A Coruña. juan.vidal.romani@udc.es

DOI:10.17979/cadlaxe.2018.40.0.4921

enviado 1/1/2018 aceptado 12/12/2018

\begin{abstract}
Since the end of the Pleistocene, 12,700 years ago, dramatic effects are taking place all along the coastline of Galicia essentially due to a continuous rise in sea level along the coast with an average rate between $3 \mathrm{~mm}$ and $3.5 \mathrm{~mm}$ per year, which means an ascent from the beginning of the Holocene of approximately 40.95 meters. The immediate effect has been a progressive reduction in the width of a continental shelf covered by beaches and extensive dune fields. The shortening of the coastal sandy strip produced a migration towards the continent of the sandy deposits that were mobilized by the waves (beaches) and / or by the wind (dunes), as it usually happens. At the present time, almost the previous maximum limit of the sea advance has been reached. The sea is located either at the base of the cliffy rocky coast or in front of freshwater bodies (rivers or coastal lagoons) that temporarily slow down the advance of the dunes or the accumulation of beach sands. Nowadays the marine action proceeds to an accelerated destruction of the beaches, where the wind excavates the storm corridors, that the sea, during the storms, will use to invade the interior. In areas of rocky coast the accumulation of sand produces the characteristic rampant or climbing dunes. In
\end{abstract}


this paper we provide some very interesting data referring to the exhumation of the levels of ancient forests and associated fauna present at the beginning of the Holocene in the will coastal strip, which now, ephemerally, are again exposed and then be destroyed definitively by the action of the wares.

Key words: Holocene dune, Holocene fossil forest, Eemien interglacial, coastal lagoon, postglacial marine transgression.

\section{Resumen}

Desde el final del Pleistoceno hace 12.700 años hasta el día de hoy se están produciendo efectos dramáticos en toda la orla litoral de la costa de Galicia debidos esencialmente al ascenso del nivel del mar en toda la costa con una velocidad media entre $3 \mathrm{~mm}$ y $3,5 \mathrm{~mm}$ anuales desde el inicio del Holoceno y que se evalúa, aproximadamente, en unos 40,95 metros. El efecto inmediato ha sido una progresiva reducción de la anchura de una plataforma continental cubierta por playas y extensos campos dunares lo que produjo una migración hacia el continente de los depósitos arenosos movilizados por las olas (playas) y/o por el viento (dunas). En el momento actual se ha alcanzado prácticamente el límite del avance transgresivo holoceno al situarse el mar en la base de la costa rocosa acantilada o en su caso frente a alguna masa de agua dulce (ríos o lagunas costeras) que frena temporalmente el avance de las dunas o la acumulación de arenas de playa. La acción marina está procediendo a la destrucción acelerada de las playas donde el viento primero excava sus corredores de tormenta que luego el mar, durante los temporales, utilizará para invadir el interior del continente. En las zonas de costa rocosa la acumulación de la arena produce las características dunas rampantes o trepadoras. En este trabajo se aportan algunos datos de gran interés referidos a la exhumación de los niveles de antiguos bosques y su fauna asociada, presentes desde el inicio del Holoceno en la franja litoral, y que ahora, efímeramente, vuelven a ser puestos al descubierto antes de ser destruidos definitivamente por la acción de las olas.

Palabras clave: dunas holocenas, bosque fósil holoceno, interglacial eemiense, lagoon costero, transgresión marina postglacial. 


\section{ANTECEDENTES}

La costa gallega se define como una costa primaria (Ottmann 1967), es decir constituida principalmente por geoformas generadas por procesos continentales. Se trata en su mayor parte de una costa rocosa (acantilados y rías) que, en menor proporción, alterna con tramos de costa arenosa (playas, lagoons y barras arenosas). Desde el comienzo de la transgresión marina holocena esta costa ha sido inundada por el mar, aunque la erosión realizada por las olas haya sido mas moderada en la costa rocosa. Por esta razón la mayor parte de las geoformas identificables ahora en la costa gallega son heredadas del pasado geológico de Galicia (Vidal Romaní 2012) y se deben a la erosión fluvial, o son consecuencia de la tectónica de placas o de movimientos diastróficos (fallas, fracturas o deslizamientos rocosos). En el resto, donde predominan las formas arenosas sucede algo similar a lo observado en la costa rocosa. Esencialmente se trata de un problema de herencia de materiales arenosos, que intervienen de forma notable en la conformación de la actual costa postglacial. Normalmente asociamos las acumulaciones de arena a formaciones playeras pero en nuestro caso la mayoría de las playas se han formado a consecuencia del retrabajado de las antiguas dunas movilizadas hacia la costa durante la transgresión holocena; en un sentido muy estricto, las únicas formaciones que podrían recibir el nombre de playas son las de cantos o shingle beach, una formación sedimentaria, por otra parte bastante frecuente en la costa gallega. (Fig. 1).

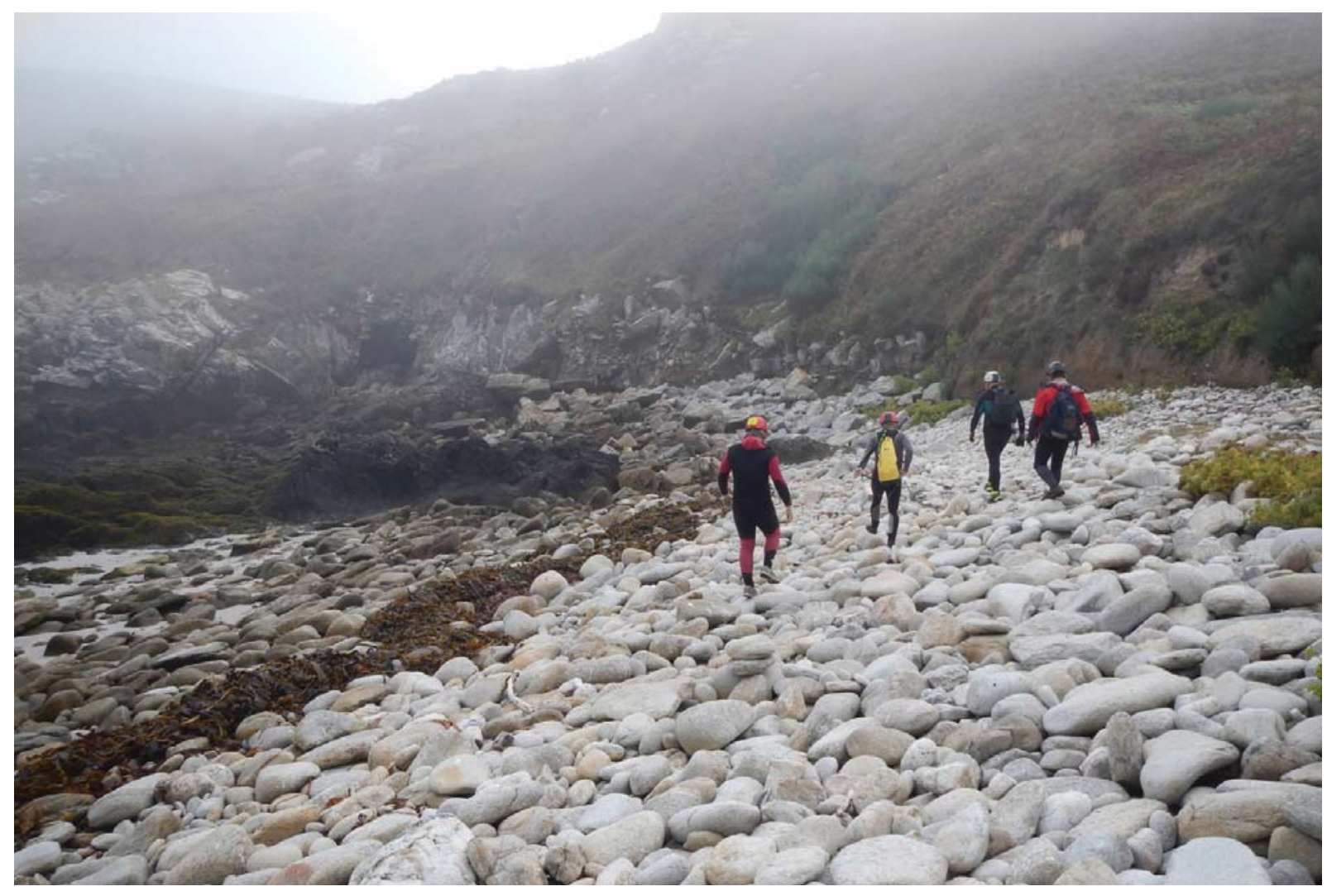

Fig. 1. Playa de cantos (shingle beach) en el extremo norte de la Isla de Ons (Pontevedra). (Foto Beatriz Bruna). 


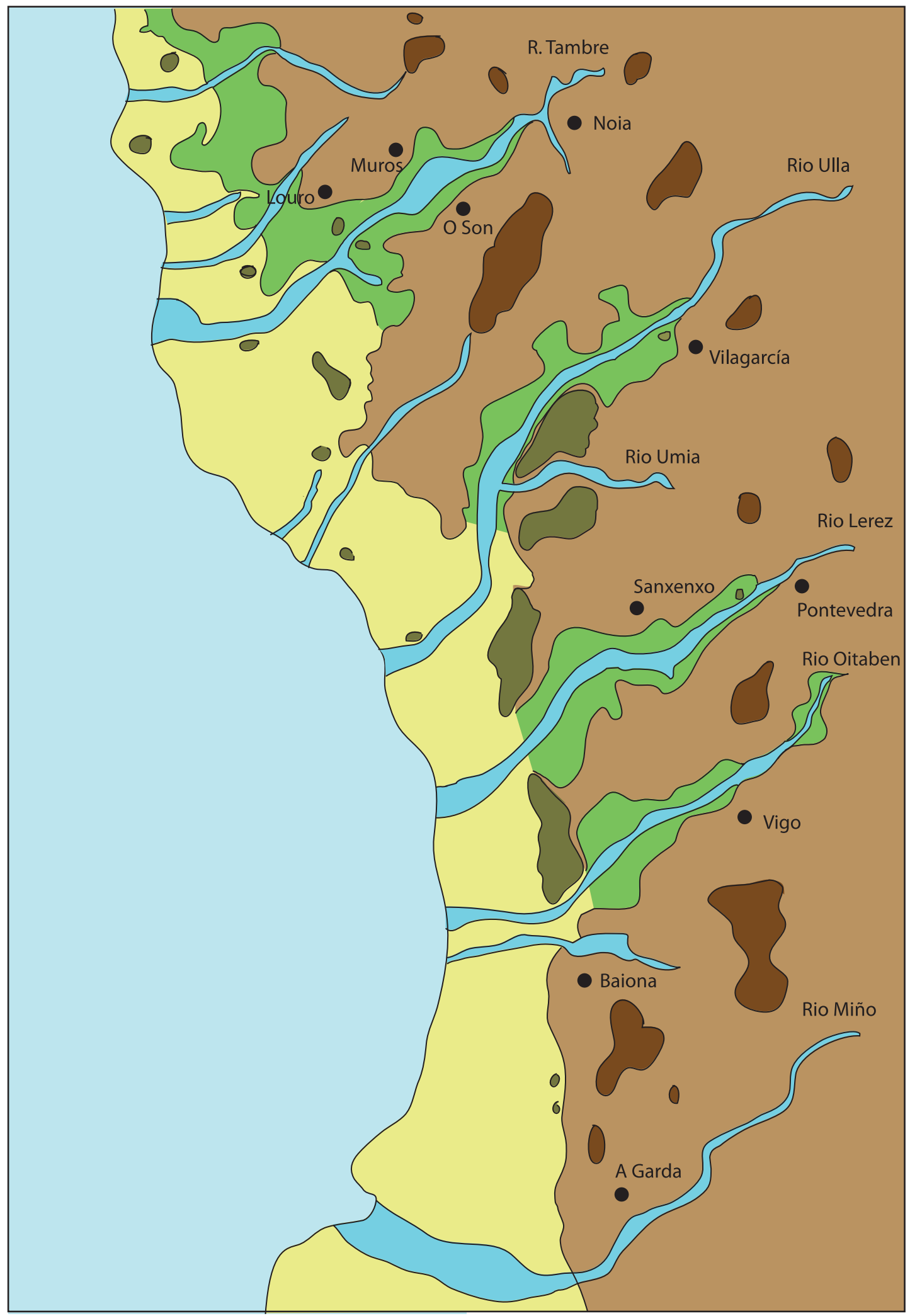

Fig. 2. Situación de la costa atlántica de Galicia al final de la última glaciación. Obsérvese la posición del nivel del mar y la franja arenosa en su máxima extensión. (Dibujo A. Grandal). 
En las zonas de desembocadura de los cursos de agua actuales tanto en el borde atlántico como en el cantábrico la costa se transformaría bien en albuferas estilo Aveiro en los casos de grandes ríos (Anllóns, Lérez, Tambre, Ulla, Verdugo, Miño, etc.) bien en lagunas de agua dulce represadas por las dunas que impedirían el progreso libre hacia la costa de los cuerpos dunares (Corrubedo, Louro, Traba, Baldaio, Doniños, Frouxeira). (Fig. 2 y 3). La totalidad de las islas actuales (Cíes, Ons, Sálvora, Sisargas) destacadas ahora del continente estarían incorporadas entonces a éste por cuerpos arenosos obviamente de origen eólico. Sin embargo al final del Pleistoceno la situación cambió radicalmente con el ascenso del nivel del mar, produciéndose un estrechamiento de la zona de playa y de la franja dunar, que fueron adelgazándose hasta transformarse en el estrecho borde arenoso actual.

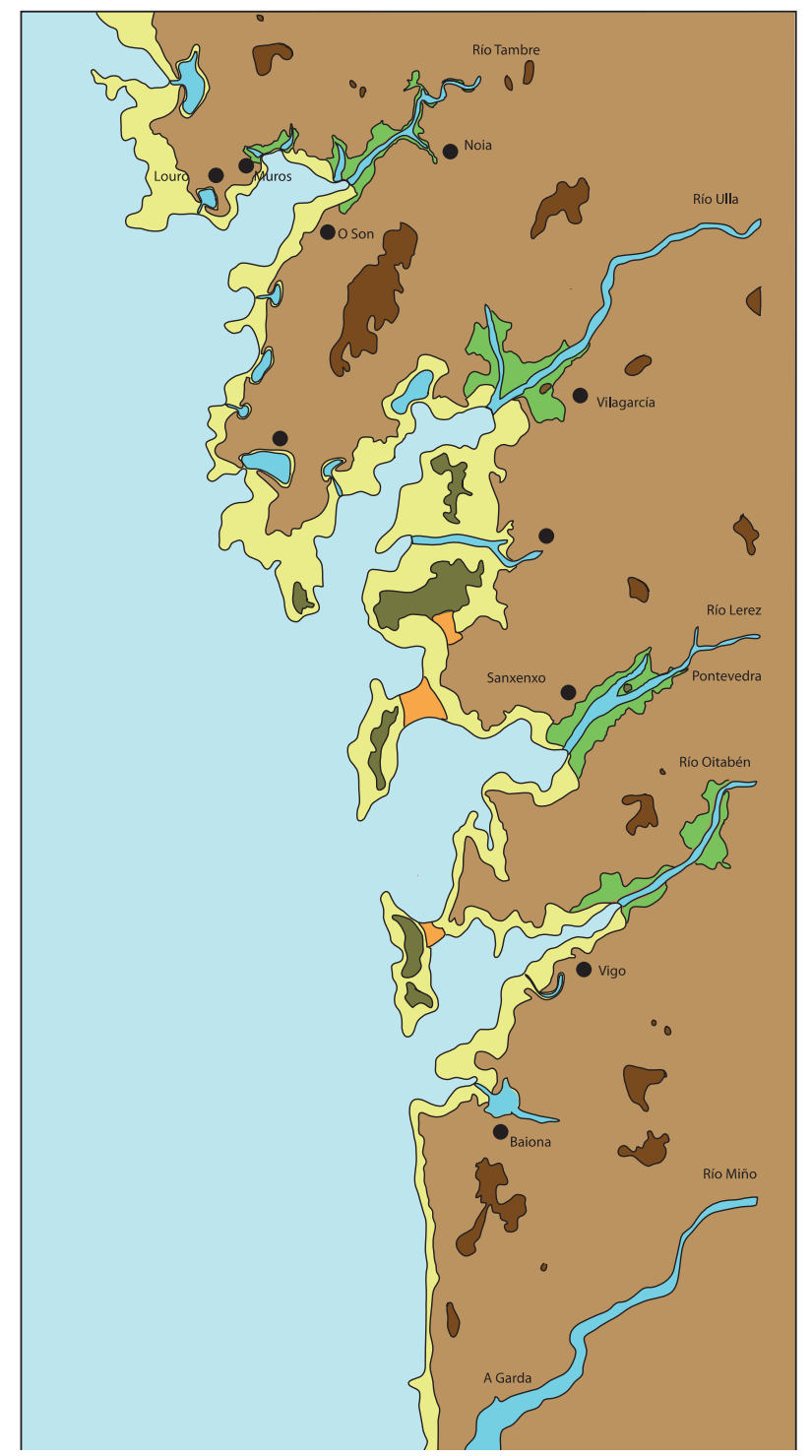

Fig. 3. Reducción de la franja arenosa (playa mas dunas) a medida que la subida del nivel del mar durante el Holoceno progresaba. (Dibujo A. Grandal). 
Desde hace 12700 años y hasta hace al menos 4000 años el aspecto de las actuales rías sería probablemente el de valles fluviales densamente poblados por la vegetación con sus ríos desembocando en una extensa zona de playa. (Fig. 2).

\section{LOS BOSQUES FÓSILES FINIPLEIS- TOCENOS Y HOLOCENOS.}

La contribución de esta nota es esencialmente presentar pruebas de la existencia de toda una orla de bosques litorales holocenos franjeando la costa de Galicia que, por tanto, no se limitaron al interior de las actuales rías (entonces valles fluviales) como hasta ahora se pensaba. En efecto, durante los últimos años, especialmente después de temporales intensos, es frecuente encontrar la mención en los medios de comunicación (prensa, radio y televisión) de niveles de bosques fósiles representados por grandes troncos, o aun tocones, enterrados en sedimentos muy finos caracterizados por un elevado contenido orgánico. (Fig. 5, 6 y 7). Todos estos hallazgos se sitúan al nivel de la marea baja equinoccial, por lo que las posibilidades de observación son muy breves e inmediatas a un período de excavación enérgico en la zona intermareal, por ejemplo después de un temporal. Por esta razón el fenómeno solo es observable brevemente, normalmente unas horas o a lo sumo uno o dos días desapareciendo luego los restos de bosques fósiles, bien por erosión marina, bien por un nuevo enterramiento en la arena movilizada por las olas.

Este tipo de hallazgos no se relaciona con las zonas mas protegidas de la costa (interior de rías) sino que, al contrario, suele darse en las zonas mas vulnerables al oleaje pues es precisamente allí donde la mayor energía de las olas pone al descubierto los antiguos bosques fósiles.

Una característica común a todos estos afloramientos es la de que el estrato arbóreo holoceno se desarrolló de forma invariable, sobre un nivel de gravas o cantos mas o menos potente. Las gravas con un diámetro de medio a grande (entre 4 y $256 \mathrm{~mm}$ de diámetros) pueden presentar diferente grado de redondeamiento y van desde subangulosas a muy bien rodadas. Normalmente debajo del nivel de gravas (Nieto y Vidal Romaní 1989), aparece una plataforma rocosa, lo que confirma que se trata de depósitos relacionados con un nivel de abrasión marina, formado probablemente durante el máximo del interglacial Eemiense (EI5e,140 ka antes de ahora) y que en el momento actual está comenzando nuevamente a ser alcanzado por el mar. (Fig. 4). 
CAD. LAB. XEOL. LAXE 40 (2018) Nota sobre la última transgresión marina en la costa de Galicia 235

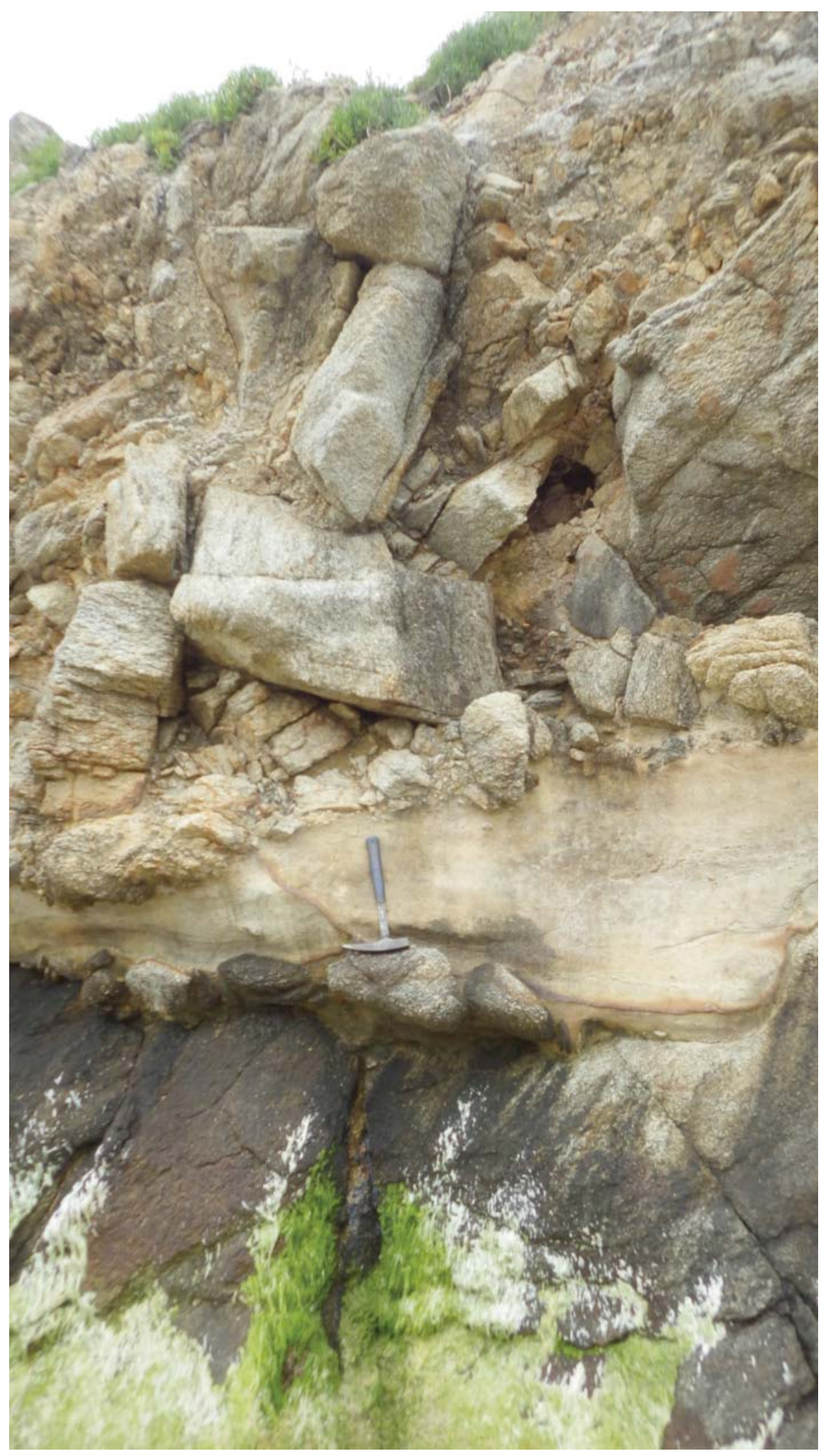

Fig. 4. Secuencia completa desde la última glaciación. En la base niveles de cantos del interglacial Eemiense. Sobre ellos la duna y los derrubios periglaciales correspondientes a la última glaciación. Representaría la sedimentación previa a la Transgresión holocena actual. (Baldaio, Coruña). 


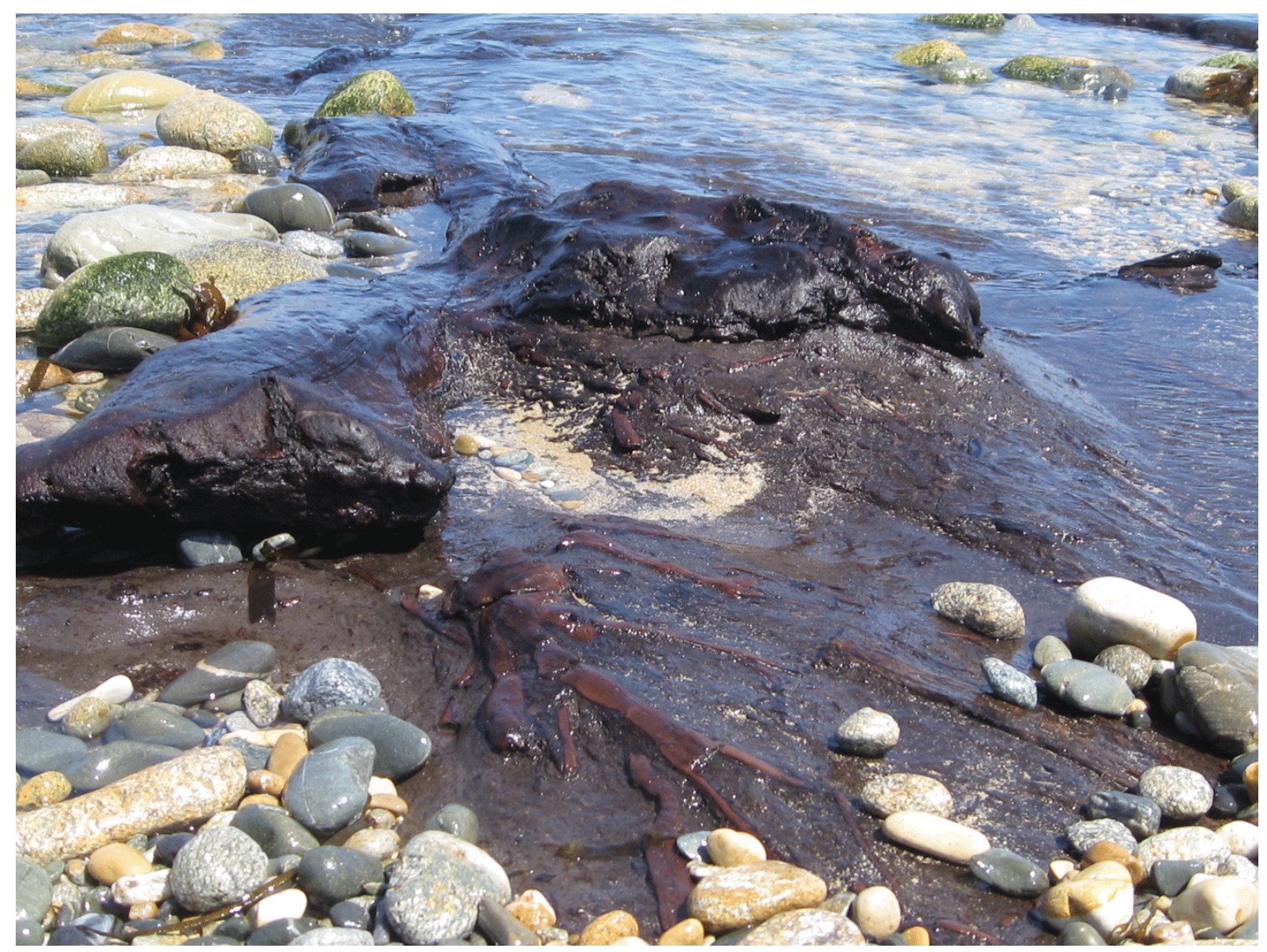

Fig. 5. Nivel de bosque holoceno (aproximadamente 7000 años antes de ahora) apoyado sobre la playa de cantos del anterior máximo interglacial del Eemiense. (Playa de Cobas, Ferrol, Coruña).

Es sobre este nivel marino de gravas, y mucho tiempo después con el mar muy alejado de la costa (episodio regresivo) sobre el que se desarrollará un bosque del que se conservan numerosos restos, desde tocones aun enraizados en la base, a troncos o ramas de diverso tamaño que aparecen enterrados entre las gravas. Hasta el momento estos restos vegetales han dado edades muy variables entre 29ka y 4kya BP (Granja y Soares de Carvalho 1995 a y b; López Cancelo 2004) y aparentemente correspondientes al Pleistoceno tardío o normalmente al Holoceno. Verosímilmente, con un nivel del mar mas bajo que el actual, podrían encontrarse restos de bosques aun mas antiguos aunque esto no ha ocurrido hasta el momento.

Los restos de bosques fósiles en la mayor parte de los afloramientos estudiados están englobados en un nivel lutítico muy orgánico interpretado como un sedimento de marisma o de lagoon de agua dulce según los datos del análisis micromorfológico y palinológico (Santos y Vidal Romaní 1992, 1993;Mosquera 2001; Mosquera et al. 1994; López Cancelo 2004; Ribeiro et al. 2011, 2014). (Fig. 1 y 5). Estos datos demuestran que estos depósitos orgánicos corresponden a una etapa posterior a la de la implantación del bosque cuando éste se ve primero atrapado 


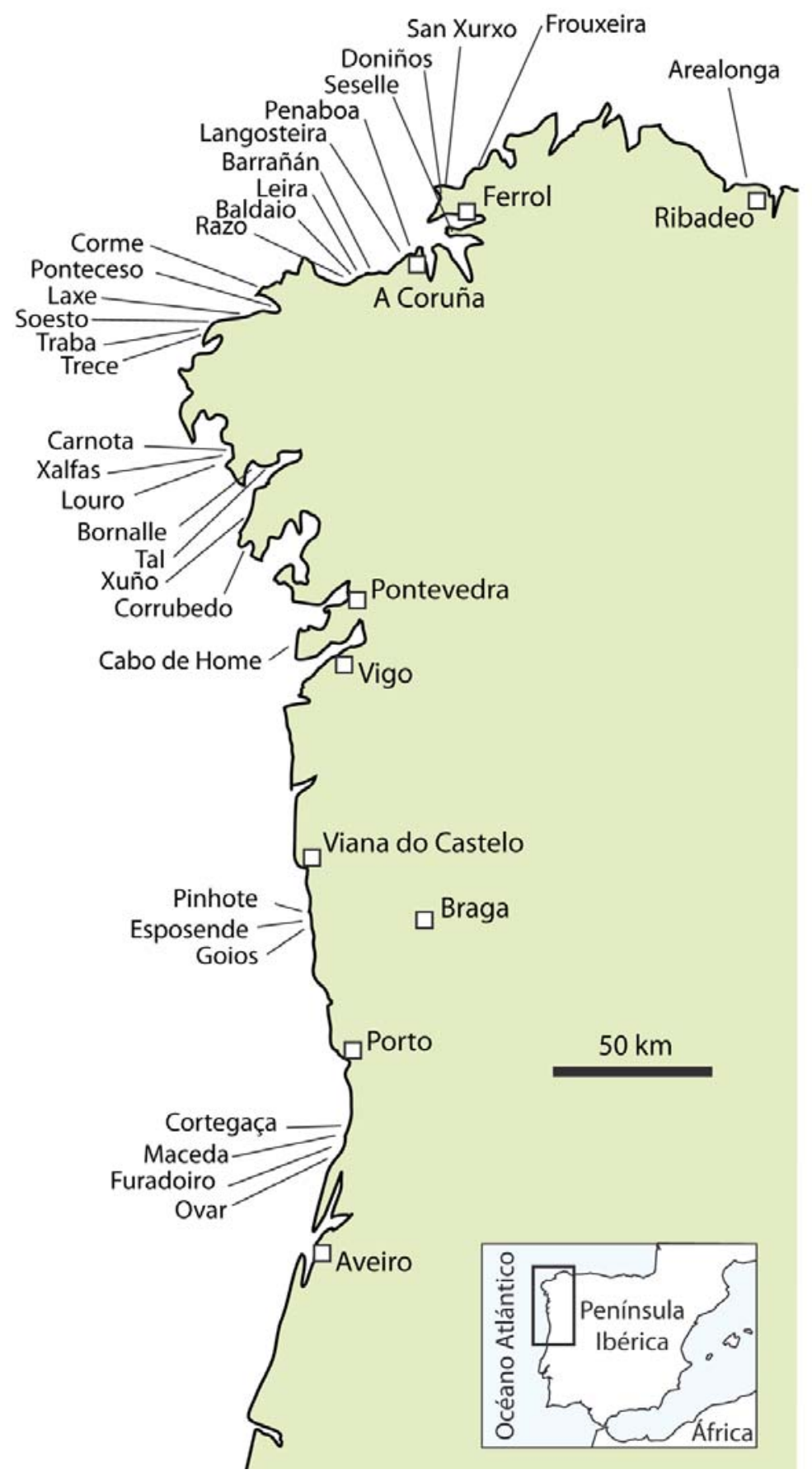

Fig. 6. Localidades en la costa atlántica-cantábrica en donde se han encontrado restos de bosques fósiles hasta el momento. Algunos de ellos son mencionados en este trabajo.

en los lagoons costeros creados por el avance de las dunas holocenas y finalmente muerto por inundación y posteriormente enterrado por las dunas. (Fig. 10). El hallazgo de restos del bosque fósil finipleistoceno-Holoceno en la costa de Galicia no es un hecho constante y a veces esta secuencia marino-continental transgresiva se simplifica conservándose 


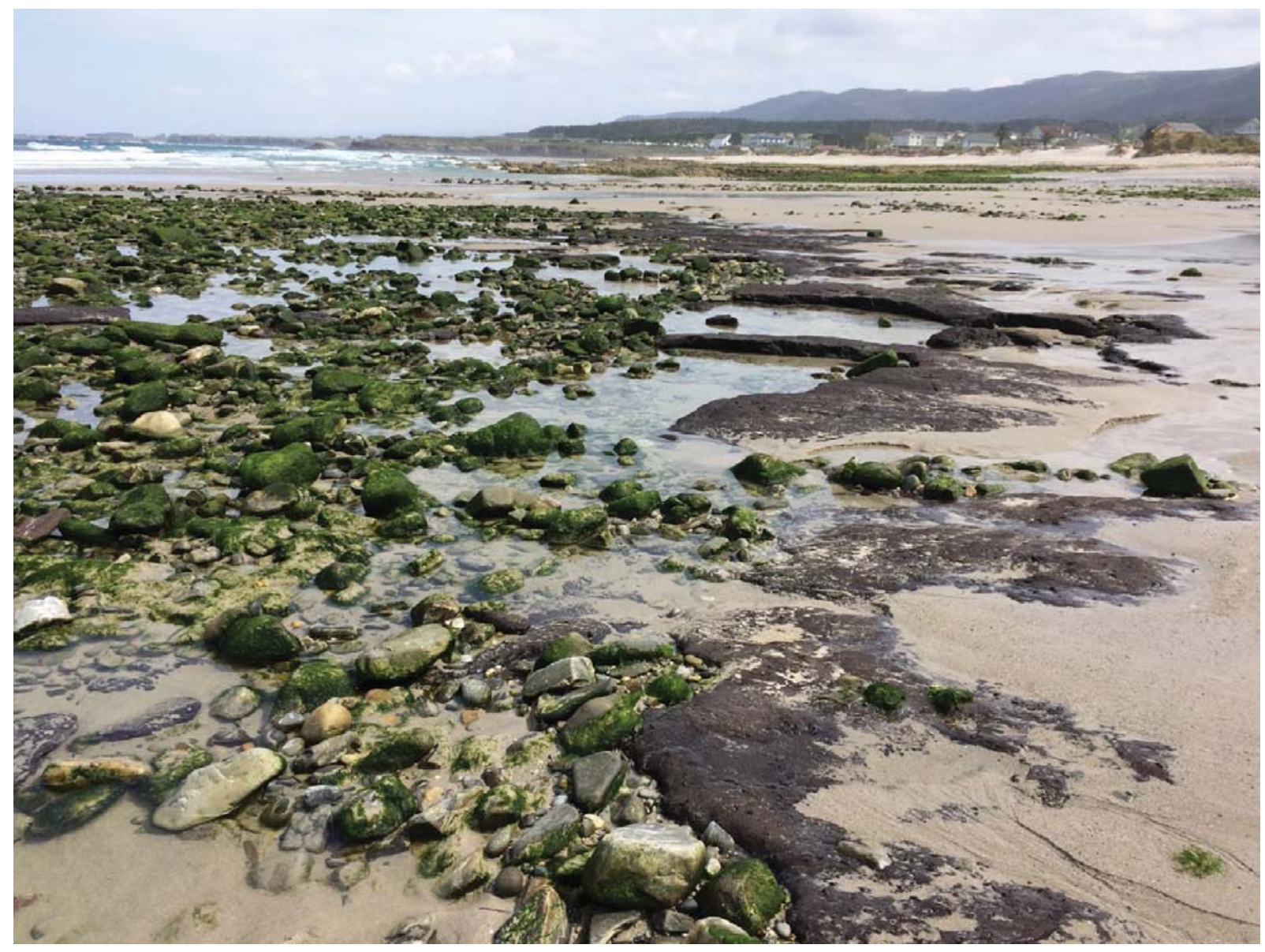

Fig. 7. Restos de los depósitos lagunales apoyados sobre la shingle beach en San Miguel de Reinante (Lugo).

únicamente el nivel lutítico orgánico (López Cancelo 2004; Ribeiro et al. 2011, 2014) aunque en todos los casos siempre está fosilizado por la duna de edad holocena (s.1.).

En algunos casos esta transición lagoon duna están muy bien marcados como ocurre en la laguna de Corrubedo (Vilas et al., 1986) y también en la ría de Muros en Tal (Arce Chamorro 2017; Gutiérrez Becker 2008).

\section{RESTOS DE VERTEBRADOS EN LOS DEPÓSITOS DE LAGOON O MARIS- MA DE AGUA DULCE.}

Aunque está fuera de toda duda que los bosques fósiles objeto de este trabajo están in situ una prueba adicional la daría encontrar algún resto de fauna asociada a ellas. Hasta el momento, sólo en dos casos se han localizado restos óseos de vertebrados. En la playa de Arealonga (Barreiros, Lugo), se recuperaron una serie de restos óseos incluidos total o parcialmente en el depósito orgánico aunque en algunos casos, existen concentraciones de huesos en surcos por debajo del límite mínimo de marea baja, (Vidal Romani y Cotelo, 2017). Entre los huesos recuperados se identifican tanto faunas silvestres y domésticas, como ciervo (Cervus elaphus), jabalí (Sus scrofa), caballo (Equus caballus) $\mathrm{y}$ vaca (Bos taurus), esta última tiene la cronología más reciente, (medieval). En Seselle 
(Ares, A Coruña), se recuperó así mismo un resto óseo incluido en el depósito orgánico, identificado como un radio de caballo, y que fue datado por 14C AMS en $3460 \pm 30$ años BP (Beta-501356).

Asimismo, y fruto de un arrastre y depósito por parte de la dinámica oceánica, en Arealonga se recuperó un resto de mamífero de talla grande que se identificó mediante huella peptídica como una Orca (figura 8).

Esta técnica consiste en la obtención de colágeno a partir del resto óseo (Pérez Rama et al 2015) y su rotura mediante la enzima tripsina, para obtener una serie de péptidos entre los cuales se encuentran algunos cuya masa/carga es característica de cada especie o género (Buckley et al. 2014).

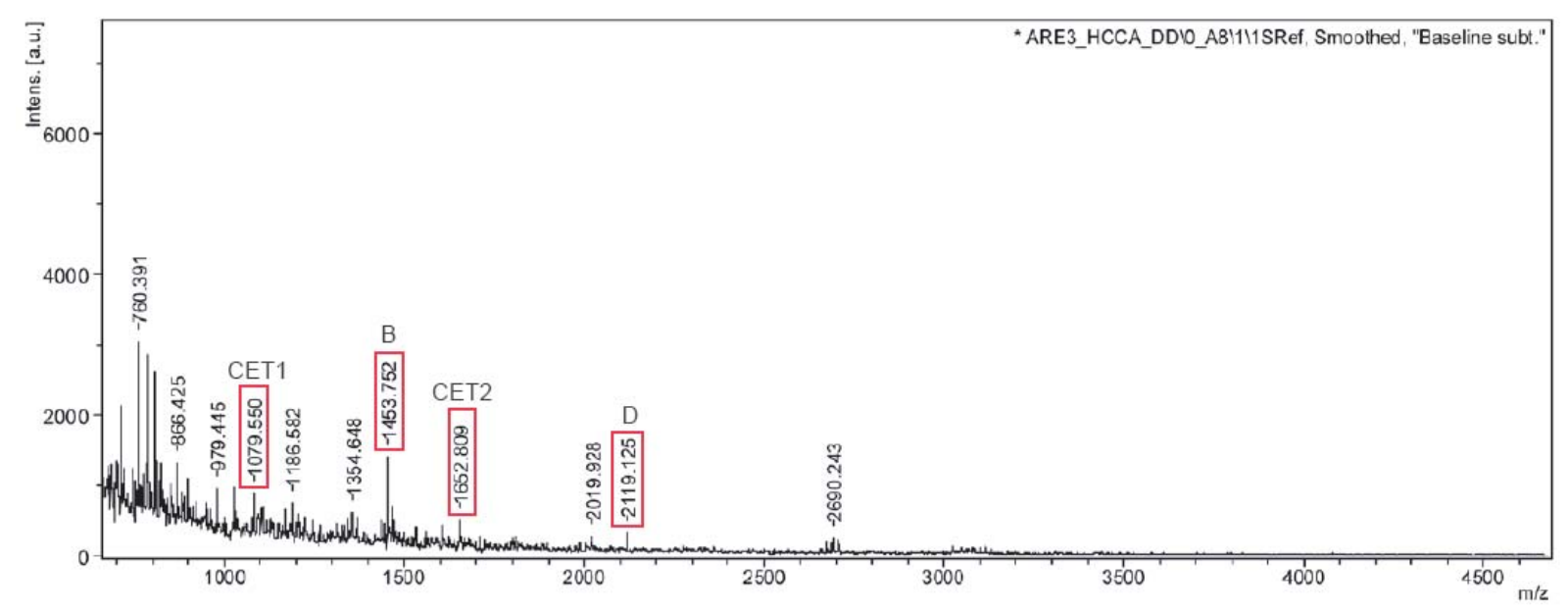

Fig. 8. Espectro de marcadores peptídicos colagénicos que permiten identificar al mamífero de gran talla de Arealonga como cetáceo odontoceto, probablemente orca (Orcinus orca).

El análisis de isótopos estables del colágeno de algunos de los mamíferos recuperados permite reconstruir su dieta y por extensión el tipo de vegetación y ambiente en el que vivieron. Los datos se presentan en la Tabla 1 y la Figura 9. En todos los casos, los valores de $\boldsymbol{\delta}^{13} \mathrm{C}$ (la proporción entre el isótopo pesado y el ligero del carbono, comparada con un estándar) son muy negativos, lo que refleja un ambiente muy arbolado en el que ocurría el llamado "efecto canopy", un escaso intercambio de gases con la atmósfera que provoca la acumulación del isótopo ligero en el sotobosque y las plantas de las que se alimentarían los herbívoros (Drucker et al 2008). Contrastan estos valores con los escasos datos contemporáneos de la Galicia interior, procedentes de la Cova do Santo en Ourense (López Costas et al 2015), o los de Cova Eirós en Lugo y Rebolal en Ourense (Grandal y Vidal 2017), cuyos valores son apreciablemente más positivos (Figura 9). Los valores de $\boldsymbol{\delta}^{13} \mathrm{C}$ negativos también indican una escasa influencia del medio marino, ya que en ambientes oceánicos la fuente de $\mathrm{C}$ para los vegetales no es sólo el $\mathrm{CO}_{2}$ atmosférico sino otros carbonatos disueltos con valores isotópicos más positivos (Chisholm et al. 2006). Esto queda bien patente en la señal isotópica del cetáceo (ver Tabla 1), claramente diferentes a la de los mamíferos terrestres. 


\begin{tabular}{|c|c|c|c|c|c|c|c|c|c|}
\hline Sitio & Especie & $\begin{array}{c}14 C \text { años } \\
\text { BP }\end{array}$ & años cal BP & $\delta^{13} C_{\text {VPDV }}(\% 0)$ & $\delta^{15} \mathbf{N}_{\mathrm{AIR}}(\% 0)$ & rend $\%$ & $\begin{array}{c}\% \mathrm{C} \\
\text { os }\end{array}$ & $\begin{array}{c}\mathbf{N} \\
\text { os } \\
\end{array}$ & $\mathrm{C} / \mathrm{N}$ at \\
\hline Arealonga & $\begin{array}{l}\text { Bóvido } \\
\text { (A) }\end{array}$ & $1530 \pm 30$ & $1525-1350$ & $-21,2$ & 6,3 & 24,4 & 42,0 & 15,2 & 3,2 \\
\hline Arealonga & $\begin{array}{l}\text { Ciervo } \\
\text { (A) }\end{array}$ & $3080 \pm 30$ & $3370-3210$ & $-21,7$ & 6,5 & 12,5 & 34,7 & 12,5 & 3,2 \\
\hline Arealonga & $\begin{array}{l}\text { Caballo( } \\
\text { A) }\end{array}$ & $3460 \pm 30$ & $3830-3641$ & $-22,1$ & 3,4 & 19,4 & 40,2 & 14,4 & 3,2 \\
\hline Arealonga & Orca (A) & $3600 \pm 30$ & $3980-3835$ & $-13,0$ & 16,7 & 15,5 & 38,5 & 13,7 & 3,3 \\
\hline Arealonga & \begin{tabular}{|l} 
Ciervo \\
(A)
\end{tabular} & $3790 \pm 30$ & $4285-4080$ & $-22,0$ & 3,8 & 15,5 & 39,9 & 14,3 & 3,2 \\
\hline Arealonga & $\begin{array}{l}\text { Jabalí } \\
\text { (A) }\end{array}$ & - & & $-22,0$ & 7,7 & 19,8 & 39,5 & 14,2 & 3,2 \\
\hline Seselle & $\begin{array}{l}\text { Caballo } \\
\text { (S) }\end{array}$ & $4190 \pm 30$ & $4840-4625$ & $-22,4$ & 4,0 & 20,6 & 38,5 & 14,2 & 3,2 \\
\hline $\begin{array}{l}\text { Cova do } \\
\text { Santo }\end{array}$ & $\begin{array}{l}\text { Ovicapri- } \\
\text { no (CS) }\end{array}$ & $4400 \pm 40$ & $5270-4855$ & $-20,3$ & 6,4 & & & & \\
\hline $\begin{array}{l}\text { Cova do } \\
\text { Santo }\end{array}$ & \begin{tabular}{|l} 
Cerdo \\
$(\mathrm{CS})$
\end{tabular} & - & - & $-20,7$ & 9,1 & & & & \\
\hline $\begin{array}{l}\text { Cova do } \\
\text { Santo }\end{array}$ & $\begin{array}{l}\text { Humano } \\
\text { (CS) }\end{array}$ & $3486 \pm 31$ & $3840-3645$ & $-19,8$ & 9,5 & & & & \\
\hline Rebolal & $\begin{array}{l}\text { Humano } \\
(\mathrm{R})\end{array}$ & $4699 \pm 41$ & $5580-5320$ & $-19,9$ & 10,0 & & & & \\
\hline Eirós & $\begin{array}{l}\text { Humano } \\
\text { (E) }\end{array}$ & $3151 \pm 31$ & $3450-3260$ & $-20,7$ & 7,7 & & & & \\
\hline
\end{tabular}

Tabla 1. Análisis isotópico de restos óseos recuperados en Arealonga (A) y Seselle (S). Además de los valores isotópicos de $\mathrm{C}$ y N, se incluyen los indicios de calidad del colageno (Ambrose 1990): rendimiento de la extracción en porcentaje, porcentajes de $\mathrm{C}$ y $\mathrm{N}$ colagénicos y relación $\mathrm{C} / \mathrm{N}$ atómica. A efectos comparativos se presentan datos de otros yacimientos contemporáneos del interior de Galicia.

La señal isotópica del nitrógeno, por su parte, se relaciona con el nivel trófico del individuo (DeNiro, 1985). Si se contemplan solamente las señales de los ungulados herbívoros, la señal reflejará la mayor o menor actividad bacteriana en los suelos, que se relaciona con el clima (Amundson et al.
2003). En los ungulados hallados en estos depósitos se observa un incremento en la señal isotópica del $\mathrm{N}$ desde los de cronología más antigua hacia los más recientes, probablemente reflejando el atemperamiento climático en esta secuencia contemporáneo al avance del postglacial holoceno. 


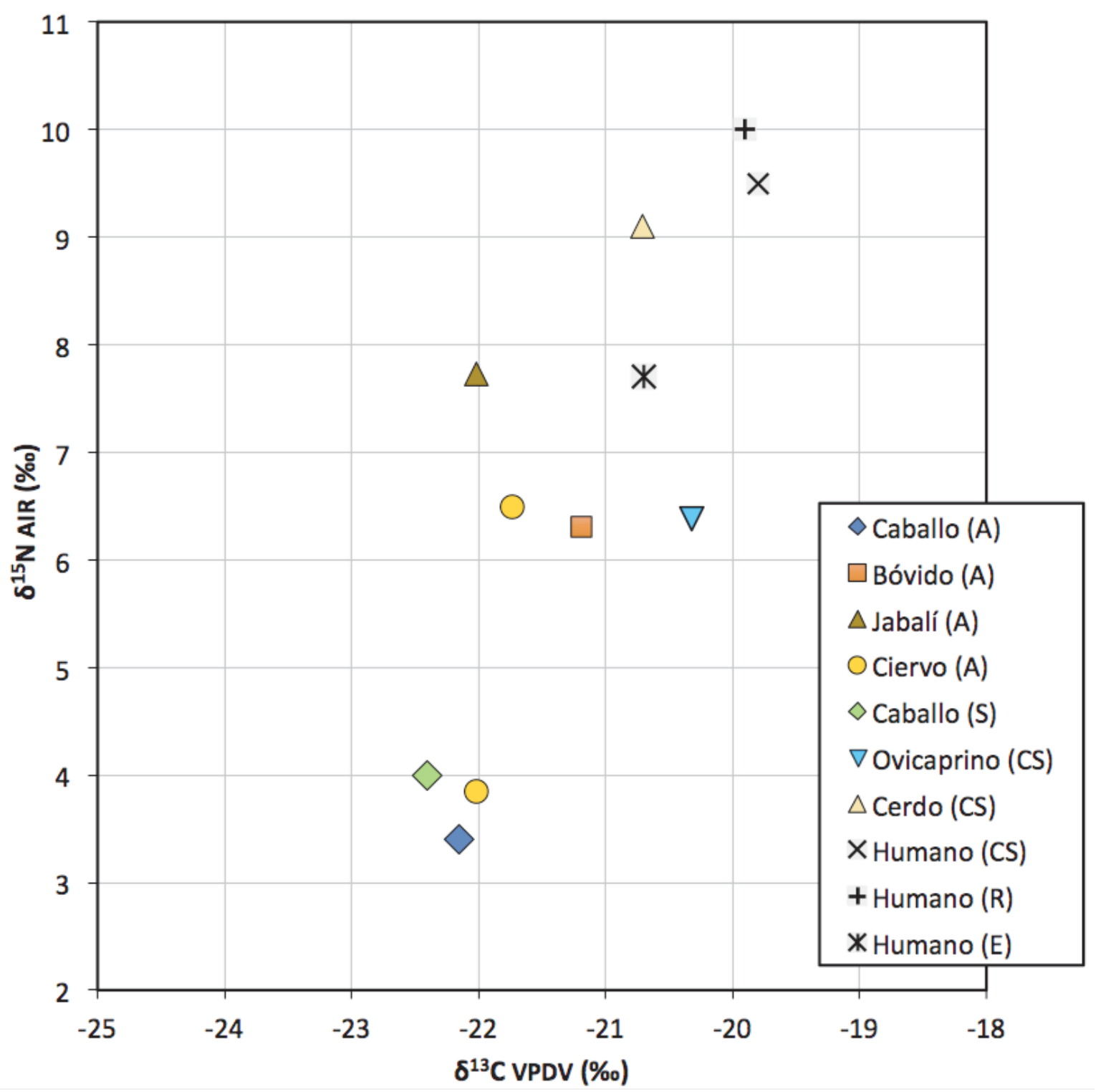

Fig. 9. Señales isotópicas del colágeno óseo de los mamíferos terrestres recuperados en los depósitos costeros de Arealonga y Seselle, comparados con otros individuos contemporáneos del interior de Galicia. Leyenda como Tabla 1.

Vemos por tanto que el estudio isotópico de estos mamíferos, en muchos casos contemporáneos con la vegetación de bosque y también con los depósitos orgánicos de lagoon e incluidos en ellos, contribuye a la reconstrucción del episodio transgresivo: un entorno de bosque, con una cubierta arbórea muy desarrollada, y escasa influencia marina que progresivamente, a medida que prosigue la subida del nivel del mar se va haciendo cada vez mas clara (Santos y Vidal Romaní 1992, 1993). Como se esquematiza en la figura 10. 


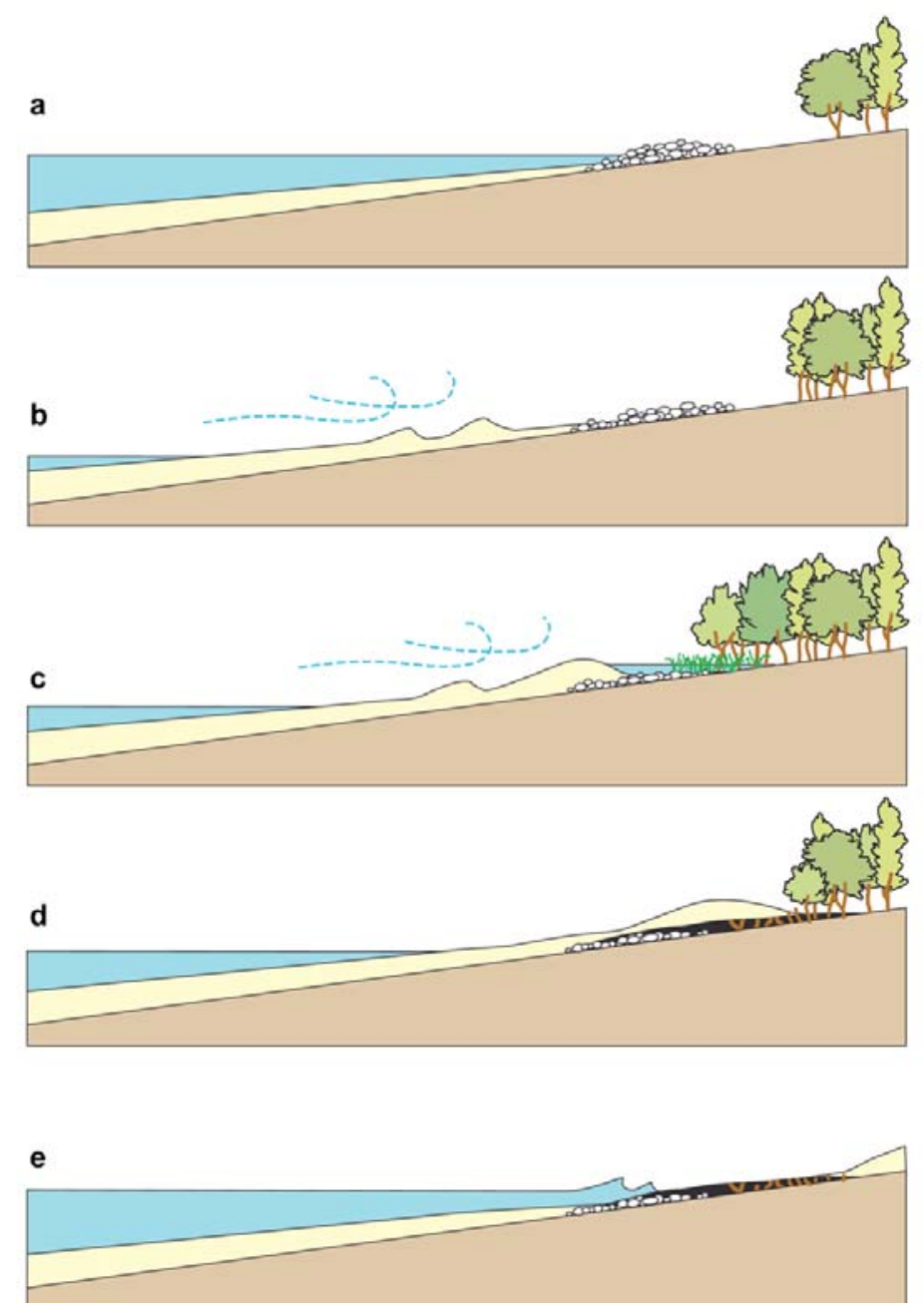

Fig. 10. Secuencia de posiciones del nivel del mar desde el máximo interglaciar eeemiense (a), al estacionamiento antes del comienzo del nivel del mar en su posición mas alejada (b). Las posiciones c, d y e, marcan la transgresión postglacial holocena hasta alcanzar la posición actual del mar. Esta secuencia puede completarse con la mostrada en la Fig. 4.

\section{CONCLUSIONES}

A pesar de la gran extensión de costa que tiene Galicia, el episodio holoceno que corresponde a los últimos 17200 años estaba, hasta ahora, muy mal documentado (López Cancelo y Vidal Romaní, 2000; López Cancelo 2004). Ello se debe, por una parte, a un desconocimiento generalizado de la cronología de los niveles marinos representados por playas de cantos (shingle beach) (Arce Chamorro 2017) y cuya cronología solo se ha estipulado, arbitrariamente, en función de su altura sobre el nivel del mar actual. Sin embargo en los últimos años (Viveen 1992 a y b; 1993 a y b) se ha puesto de manifiesto la existencia de movimientos neotectónicos, muy activos desde, al menos, el Cenozoico final que están elevando la costa gallega aceleradamente lo que descarta la validez de una correlación entre niveles marinos basada únicamente en su altura sobre el nivel del mar y exigiendo una datación por técnicas de O.S.L., (Arce Chamorro 2017). Lo mismo 
ocurre con los depósitos arenosos costeros que han sido identificados invariablemente como niveles de playa (Costas Otero 2006; Martínez Carreño 2015) y datados, cuando era posible, por radiocarbono o en su caso por su altura sobre el nivel del mar actual. Raramente, o nunca, se han identificado estos depósitos como eólicos (Gutiérrez Becker et al. 2004; Gutiérrez Becker 2008; Arce Chamorro 2017; Trindade et al. 2013). Consideramos que por ejemplo una duna rampante o trepadora no puede ser datada por su cota topográfica pues, con la misma edad absoluta, una duna alcanza alturas muy diferentes por encima del nivel del mar. Por eso interpretar secciones de una misma duna como distintos niveles marinos altera totalmente el significado de la unidad sedimentaria ignorando que corresponde a un único episodio eólico de regresión marina y no a un estacionamiento del mar durante una etapa marina transgresiva a distintas alturas. Errores como estos, lamentablemente frecuentes, han conseguido desvirtuar en gran medida los datos conocidos sobre esta etapa evolutiva de la costa correspondiente al Holoceno en la costa gallega. Sin embargo el escenario descrito en nuestro trabajo, basado en datos reales, refleja un paisaje costero enormemente cambiante durante el cual la costa gallega cambia radical y aceleradamente su morfología, primero por la invasión de la costa por las dunas y luego por la inundación de los valles fluviales por el mar, induciendo grandes cambios tanto en la flora como en la fauna, incluida obviamente la especie humana y sus hábitats costeros. La costa gallega, que durante la última fase glacial, mas de 100.000 años, se había mantenido intacta se ha visto modificada aceleradamente en menos de 7000 años.

\section{AGRADECIMIENTOS:}

Los autores agradecen a la asociación Mariña Patrimonio y a D. David Cobelo la entrega de los restos fósiles para su estudio.

\section{BIBLIOGRAFÍA}

Ambrose SH (1990) Preparation and characterization of bone and tooth collagen for stable carbon and nitrogen isotope análisis. Journal of Archaeological Science 17: 431-451.

Amundson R, Austin AT, Schuur EAG, Yoo K, Matzek V, Kendall C, Uebersax A, Brenner D, Baisden WT (2003) Global patterns of the isotopic composition of soil and plant nitrogen. Global Biogeochemical Cycles, 17:1031.

Arce Chamorro C (2017) Datación por luminiscencia de depósitos fluviales y eólicos en el margen occidental de Galicia. Tesis Doctoral. Universidade de A Coruña.

Bao R, Vidal-Romaní JR, Varela M (1993) Fossil diatoms from surface sediments in the Galician Rías and the continental shelf (NW Spain): Preliminary results. III ème Colloque International d'Océanographie du Golfe de Gascogne. Actas, 185-189.

Bao R, Vidal-Romaní JR, Varela M (1993) Diatomeas dulceacuícolas y episodios regresivos en el margen continental de Galicia. Actas $3^{\text {a }}$ Reunião do Quaternario Ibérico. Coimbra, 237-240.

Buckley M, Fraser S, Herman J, Melton ND, Mulville J, Pálsdóttir AH (2014) Species identification of archaeological marine mammals using collagen fingerprinting. Journal of Archaeological Science 41:631-641 
Chisholm BS, Nelson DE, Schwarcz HP (2006) Stable carbon isotope ratios as a measure of marine versus terrestrial protein in ancient diets. Science 216, 11311132.

Costas Otero S (2006) Evolución morfosedimentaria anual, decadal y secular del sistema barrera lagoon de Cíes durante el Cuaternario (Galicia, NO Península Ibérica). Tesis Doctoral. Universidad de Vigo. 214 pp.

De Niro MJ (1985) Post-mortem preservation and alteration of in vivo bone collagen isotope ratios in relation to paleodietary reconstruction. Nature 317: 806-809.

Drucker D, Bridault A, Hobson K, Szuma E, Bocherens H (2008) Can carbon-13 in large herbivores reflect the canopy effect in temperate and boreal ecosystems? Evidence from modern and ancient ungulates. Palaeogeography, Palaeoclimatology, Palaeoecology 195:375-388.

Gómez Orellana L (2002). El último ciclo glaciar-interglaciar en el litoral del $N W$ Ibérico: dinámica climática y paisajística. Tesis Doctoral. Universidad de Santiago, 254 pp.

Granja H, Soares de Carvalho G (1995a) Is the coasline "protection" of Portugal by hard engineering structures effective?. Journal of Coastal Research, 11(4):1229-1941.

Granja H, Soares de Carvalho G. (1995b) Sea level changes during the PleistoceneHolocene in the NW coastal zone of Portugal. Terra Nova, 7(1): 60-67.

Gutiérrez Becker L (2008) Caracterización de los sistemas dunares costeros del $N W$ Ibérico y su evolución durante el Cuaternario superior. Tesis Doctoral. Universidade de A Coruña.

Gutiérrez Becker L, López-Cancelo L, Vidal
Romaní JR, Granja H. (2004) Episodios eólicos durante el Pleistoceno superiorHoloceno de la costa de Galicia. El caso de Punta Penaboa (A Coruña). Contribuciones recientes sobre Geomorfología (Vol 1). pp. 253-262.

López Cancelo L (2004). Cambios paleoambientales en el NO Peninsular durante el Holoceno a partir del estudio de foraminíferos bentónicos. Tesis Doctoral. Universidade de A Coruña.

López Cancelo L, Vidal-Romaní JR (2000) Cronología de la transgresión holocena en la Ría de Foz (Lugo, Galicia, España). Geogaceta, 87-88.

López Costas O, Müldner G, Martínez Cortizas A (2015) Diet and lifestyle in Bronze Age Northwest Spain: the collective burial of Cova do Santo. Journal of Archaeological Science 55:209-218.

Martínez Carreño N (2015) Análisis multidisciplinar de las acumulaciones de metano en relación con la arquitectura estratigráfica y los cambios del nivel del mar durante el Cuaternario en la Ría de Vigo. Tesis Doctoral. Universidade de Vigo.

Mosquera MJ, Mateu G, Vidal Romaní JR (1994) Estudio del depósito de Puerta Real. Un episodio regresivo holoceno en la Ria de A Coruña. Gaia, 9:75-78.

Mosquera Santé MJ (2001) Evolución postglacial del nivel del mar en el $N W$ de la Península Ibérica. El caso del Golfo Artabro (A Coruña, Galicia, España). Tesis Doctoral. Universidade de A Coruña.

Nieto M, Vidal Romaní JR (1989) Niveles marinos y depósitos continentales antiguos en el borde costero entre Cabo Prior y Cabo Prioriño. (A Coruña, Galicia, España). Cadernos do Laboratorio Xeolóxico de Laxe, 14: 67-78. 
Ottmann FC (1967) Introduccion a la geología marina y litoral. Editorial EUDEBA, 287 pp.

Pérez Rama M, Vaqueiro Rodríguez $M$, Grandal d'Anglade A (2015) Indicios de pastoreo extensivo en el Noroeste peninsular durante el dominio Suevo. Cadernos do laboratorio Xeolóxico de Laxe 38:107-134.

Ribeiro H, Bernal A, Flores D, Pissarra J, Abreu I, Vidal Romani JR, Noronha F (2011). A multidisciplinary study of an organic-rich mudstone in the Middle Holocene on the Northern coast of Portugal. Comunicações Geológicas, 98: 93-98.

Ribeiro H, Pinto De Jesus A, Oliveira F, Vidal Romani JR, Abreu I, Noronha F (2014). A Estudo da "Formação ArenoPelítica" na zona Litoral Porto - Vila Nova de Gaia. Contribuição do conteúdo polínico. Comunicações Geológicas, 101:631-634.

Santos ML, Vidal Romaní JR (1992) El lagoon de Seselle: un episodio de la transgresión holocena en la Ría de Ares (A Coruña, Galicia, España). Datos geomorfológicos, sedimentarios y paleoecológicos. Cadernos do Laboratorio Xeolóxico de Laxe, 18:163-174.

Santos ML, Vidal Romaní JR (1993) La transgresión holocena en la Ria de Ares (A Coruña, Galicia, España). Datos cronológicos, sedimentarios y geomorfológicos. Actas $3^{\mathrm{a}}$ Reunião do Quaternario Ibérico. Coimbra. pp. 339-345.

Trindade M J, Prudencio MI, Sanjurjo Sánchez J, Vidal-Romani JR, Ferrez T, Fernández Mosquera D, Dias M.I (2013) Post-depositional processes of elemental enrichment inside dark nodular masses of an ancient aeolian dune from
A Coruña Northwestern Spain . Geologica Acta, 2:231-244.

Vidal-Romani JR (2012). Xeoloxía, Geología, Geology. Gallaecia Petrea (Eds. Macías B. \& Guajardo, ISBN: 978-1608776-563-8). Chapter 1, 22-95. Editorial Cidade da Cultura, Santiago de Compostela.

Vidal Romani JR, Cotelo T (2017) Estudio de las posibles consecuencias del ascenso del nivel del mar en los procesos erosivos de los acantilados en el Monumento natural de la playa de "As Catedrais". Informe Geológico- Geomorfológico. 75 pp. Xunta de Galicia, Consellería de Ordenación del Medio Ambiente y Ordenación del Teritorio. Santiago de Compostela.

Vilas F, Arche A, Ramos A, Sopeña A, Rey L, Nombela P (1986) El complejo de playa-lagoon de Corrubedo y los submedios característicos. Galicia, NW. España. Acta Geológica Hispánica, T. 21-22:233-243.

Viveen W, Braucher R, Bourlès D, Schoorl JM, Veldkamp A, Van Balen RT, Wallinga J, Fernández-Mosquera D, VidalRomaní JR, Sanjurjo-Sánchez J (2012 a) A $0.65 \mathrm{Ma}$ chronology and incision rate assessment of the NW Iberian Miño River terraces based on $10 \mathrm{Be}$ and luminescence dating. Global and Planetary Change. 94-95:82-100.

Viveen W, Van Balen RT, Schoorl JM, Veldkamp A, Temme AJAM, Vidal-Romaní JR (2012 b) Assessment of recent tectonic activity on the NW Iberian Atlantic Margin by means of geomorphic indices and field studies of the Lower Miño River terraces. Tectonophysics, 544-545:13-30. 
Viveen W, Schoorl J, Veldkamp A, Van Balen R, Vidal-Romani, JR (2013 a) Fluvial terrace map of the northwest Iberian lower Miño River. Journal of Maps 9: 513-522.

Viveen W, Schoorl JM, Veldkamp A, Van Balen RT, Desprat S, Vidal-Romaní JR
(2013 b) Reconstructing the interacting effects of base level, climate and tectonic uplift in the Lower Miño River terrace record: a gradient modelling evaluation. Geomorphology, 186:96-118. 